\title{
Recognizing generalized Petersen graphs in linear time
}

\author{
Matjaž Krnc ${ }^{1}$ and Robin J. Wilson ${ }^{2}$ \\ ${ }^{1}$ University of Primorska, Slovenia, and University of Salzburg, Austria. \\ E-mail: matjaz.krnc@upr.si. \\ ${ }^{2}$ The Open University, Milton Keynes, and the London School of Economics, UK. \\ E-mail: r.j.wilson@open.ac.uk.
}

\begin{abstract}
By identifying a local property which structurally classifies any edge, we show that the family of generalized Petersen graphs can be recognized in linear time.
\end{abstract}

The generalized Petersen graphs, introduced by Coxeter [7] and named by Watkins [19], are cubic graphs formed by connecting the vertices of a regular polygon to the corresponding vertices of a star polygon. Various aspects of their structure have been extensively studied. Examples include identifying generalized Petersen graphs that are Hamiltonian [1, 2, 5, hypo-Hamiltonian [3], Cayley [14, 17], or partial cubes [11], and finding their automorphism group [8] or determining isomorphic members of the family [15]. Additional aspects of the mentioned family are well surveyed in [6, 9, while notable recent advances in the field can be found in [4, 10, 12, 13, 16, 18, 20,

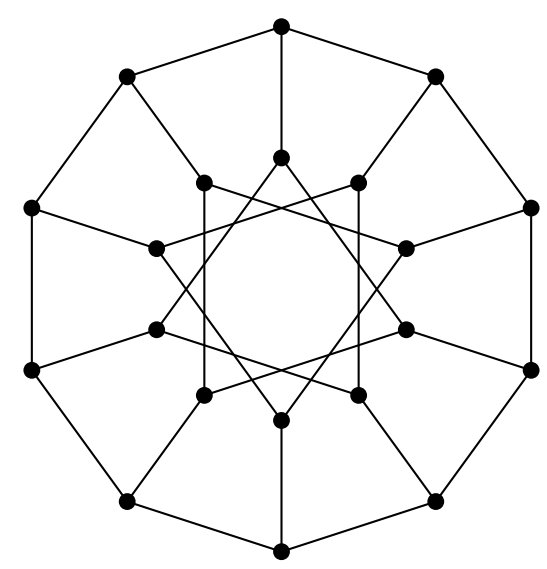

Figure 1: The generalized Petersen graph $G(10,3)$, also known as the Desargues graph.

In this paper we give a linear-time recognition algorithm for the family of generalized Petersen graphs. In particular, we identify a local property which structurally classifies any edge, whenever 
our graph is a generalized Petersen graph. We start by providing the necessary definitions and the analysis of 8-cycles; after the preliminaries in Section 1 we describe 8-cycles in Section 2. In Section 3 we introduce our main lemma, and describe and analyze the recognition procedure. At the end we mention how our Algorithm 2 behaves when the input graph is small.

\section{Preliminaries}

We follow the notations of Watkins [19] for the family GP of generalized Petersen graphs. For given integers $n$ and $k<n / 2$, we define the generalized Petersen graph $G(n, k)$ as the graph with vertex-set $\left\{u_{0}, u_{1}, \ldots, u_{n-1}, w_{0}, w_{1}, \ldots, w_{n-1}\right\}$ and edge-set $\left\{u_{i} u_{i+1}\right\} \cup\left\{u_{i} w_{i}\right\} \cup\left\{w_{i} w_{i+k}\right\}$, for $i=0,1, \ldots, i-1$, where all subscripts are taken modulo $n$. We partition the edges of $G(n, k)$ as

- the edges $E_{J}(G)$ from the outer rim (of type $u_{i} u_{i+1}$ ) which form a cycle of length $n$;

- the edges $E_{I}(G)$ from the inner rims (of type $w_{i} w_{i+k}$ ) inducing $\operatorname{gcd}(n, k)$ cycles of length $n / \operatorname{gcd}(n, k)$;

- the spokes $E_{S}(G)$ (of type $u_{i} w_{i}$ ) which correspond to a perfect matching in $G(n, k)$.

For each edge $e \in E(G)$, let $\sigma_{G}(e)$ be the number of 8-cycles containing $e$, and let $\mathcal{P}_{G}$ be a partition of the edge-set of $G$, corresponding to the values of $\sigma_{G}(e)$, for $e \in E(G)$. The mapping $\sigma$ plays a crucial role in the structural classification of edges in Section 2 for example, any spoke $e$ of the Petersen graph $G(5,2)$ has the value $\sigma_{G(5,2)}(e)=8$ (see Figure 2 ).
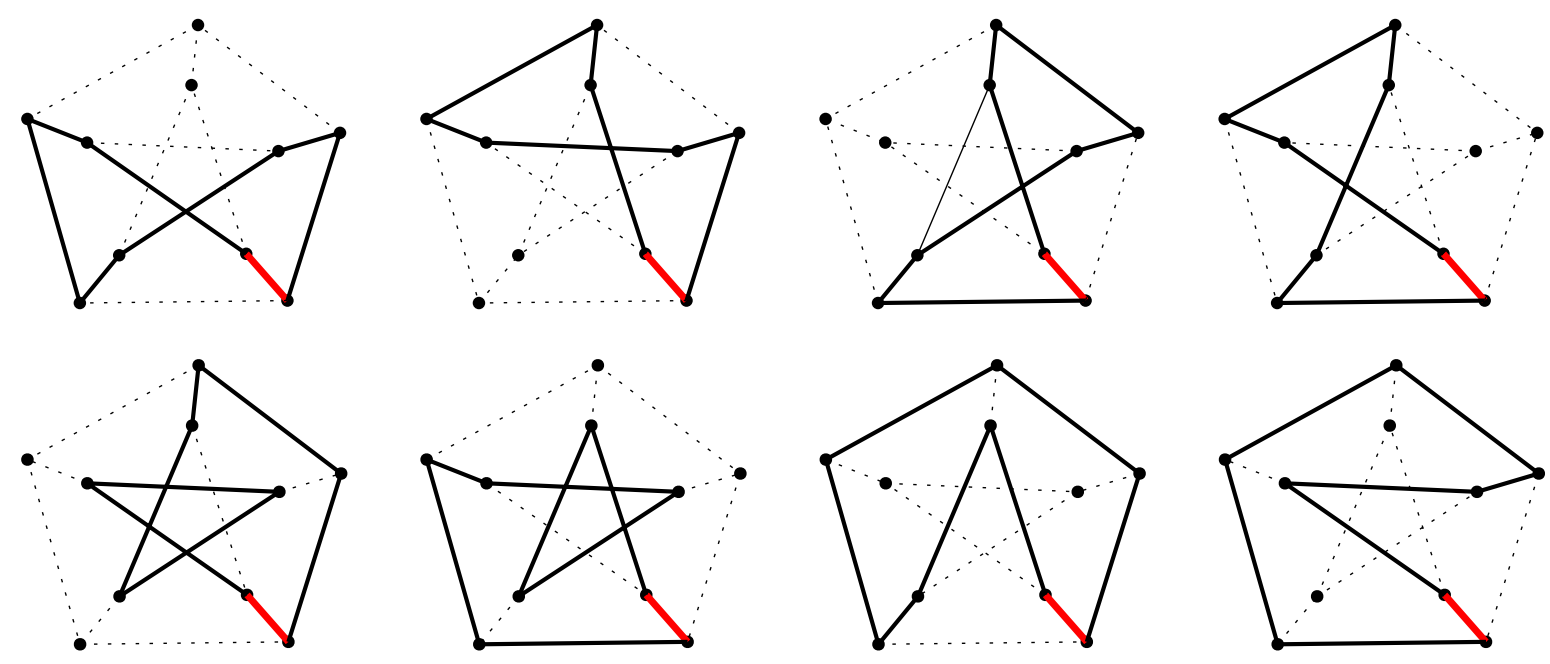

Figure 2: The Petersen graph $G(5,2)$ admits 8-cycles of types $\mathcal{C}_{2}, \mathcal{C}_{4}$, and $\mathcal{C}_{7}$, and any of its spokes $e$ has the value $\sigma_{G(5,2)}(e)=8$.

The rotational symmetry of generalized Petersen graphs implies the following remark:

Remark 1 . The edges within any of the sets $\left\{E_{J}(G), E_{S}(G), E_{I}(G)\right\}$ share the same value of $\sigma_{G}$. 
For any $G \in \mathrm{GP}$, we define $\sigma_{j}$ to be $\sigma_{G}(e)$, where $e$ is a member of $E_{J}(G) ; \sigma_{s}$ and $\sigma_{i}$ are defined similarly.

Given a graph $G$, we use the standard neighborhood notations for vertices $N_{k}(v)=\{w \in V(G) \mid$ $\left.d_{G}(v, w)=k\right\}$ or edges $N_{k}(e)=\left\{e^{\prime} \in E(G) \mid d_{L(G)}\left(e, e^{\prime}\right)=k\right\}$, where $L(G)$ is the line graph of $G$ and $d_{G}(v, w)$ is the distance between $v$ and $w$ in $G$. The notation $G[S]$ corresponds to the subgraph of $G$ induced by $S$, where $S \subseteq V(G)$ or $S \subseteq E(G)$. Graphs of order at most 80 are considered to be small graphs, and can clearly be recognized in constant time, so in what follows we assume that our graphs are not small - that is, are large. In this paper we study the large members of GP with respect to their values of $\sigma_{j}, \sigma_{s}, \sigma_{i}$, and in particular determine that such a triple can correspond to only one of eight possibilities, described below in (1). We proceed with the description of the possible 8-cycles in $G(n, k)$.

\section{A description of 8-cycles}

Given a graph $G \simeq G(n, k)$, fix a partitioning of $E(G)$ into $E_{J}(G), E_{S}(G)$, and $E_{I}(G)$, as above. Define the rotation $\rho$ on $V(G)$ by $\rho\left(u_{i}\right)=u_{i+1}$ and $\rho\left(w_{i}\right)=w_{i+1}$, for $i \in[0, n-1]$. Note that $\rho \in \operatorname{Aut}(G)$, so (non)edges are mapped to (non)edges.

We call two 8-cycles equivalent whenever we can map one into another by using rotations only. For a fixed 8-cycle $C$, we say that it has $c$-fold rotational symmetry if there exist precisely $c$ cycles equivalent to $C$ in $G$. Considering this number $c$, and also the number of edges in $E_{J}(G), E_{S}(G), E_{I}(G)$, we can explicitly describe the contributions of $C$ to the global values $\sigma_{j}, \sigma_{s}$, and $\sigma_{i}$. These contributions uniquely describe the type of cycle $C$, and are denoted by $\delta_{j}, \delta_{s}$, and $\delta_{i}$, respectively. Whenever triples $\left(\delta_{j}, \delta_{s}, \delta_{i}\right)$ are used in this paper, the corresponding cycles are always explicitly determined, or are clear from the context. We name eight specific combinations of these triples $\left(\delta_{j}, \delta_{s}, \delta_{i}\right)$ in Table 1 below. For an example of the above notions, see the eight cycles in Figure 2, which may be partitioned into three non-equivalent classes, corresponding to the types $\mathcal{C}_{2}, \mathcal{C}_{4}$, and $\mathcal{C}_{7}$.

\begin{tabular}{|c||c|c|c|c|c|c|c|c|}
\hline label & $\mathcal{C}_{0}$ & $\mathcal{C}_{1}$ & $\mathcal{C}_{2}$ & $\mathcal{C}_{3}$ & $\mathcal{C}_{4}$ & $\mathcal{C}_{5}$ & $\mathcal{C}_{6}$ & $\mathcal{C}_{7}$ \\
\hline$\delta_{j}$ & 0 & 1 & 2 & 3 & 4 & 5 & 1 & 2 \\
\hline$\delta_{s}$ & 0 & 2 & 2 & 2 & 2 & 2 & 2 & 4 \\
\hline$\delta_{i}$ & 1 & 5 & 4 & 3 & 2 & 1 & 1 & 2 \\
\hline
\end{tabular}

Table 1: The labeling of all 8 specific types of 8 -cycles in $G(n, k)$ is uniquely defined by the corresponding values of $\left(\delta_{j}, \delta_{s}, \delta_{i}\right)$. By characterizing 8-cycles later we will see that no other type of 8-cycle may appear.

If an 8-cycle $C$ uses $s$ edges from $E_{S}(G)$, we say that $s$ is the spoke value of $C$. Note that the spoke value is always 0,2 , or 4 , and that the spoke value coincides with $\delta_{s}$ only when the rotational symmetry of $C$ is $n$-fold. For the eight specific triples of $\delta_{j}, \delta_{s}$, and $\delta_{i}$ we name the corresponding eight types of such cycles by $\left\{\mathcal{C}_{i}\right\}_{i=0}^{7}$, as described in Table 1 . We will see later that no other types are possible for the generalized Petersen graphs.

We proceed by listing each of the possible 8-cycles, giving its spoke values $s$, and describing its rotational symmetries, the existence conditions, and the corresponding values of $\delta_{j}, \delta_{s}$, and $\delta_{i}$. We summarize these results in Table 2. Watkins [19] has made a list of 8-cycles for generalized Petersen graphs, similar to our Table 2 , but he did not explain why his list is complete. 


\begin{tabular}{|c|c|c|}
\hline type & a representative of an 8-cycle & existence conditions \\
\hline \hline $\mathcal{C}_{0}$ & $\left(w_{0}, w_{k}, \ldots, w_{7 k}\right)$ & $n=8 k$ or $3 n=8 k$ \\
\hline $\mathcal{C}_{1}$ & $\left(u_{0}, w_{0}, w_{k}, w_{2 k}, w_{3 k}, w_{4 k}, w_{5 k}, u_{5 k}\right)$ & $n=5 k \pm 1$ or $2 n=5 k \pm 1$ \\
\hline \multirow{2}{*}{$\mathcal{C}_{2}$} & $\left(u_{0}, u_{1}, u_{2}, w_{2}, w_{2+k}, w_{2+2 k}, w_{2+3 k}, w_{2+4 k}\right)$ & $n=4 k+2$ or $2 n=4 k+2$ \\
\cline { 2 - 3 } & $\left(u_{2}, u_{1}, u_{0}, w_{0}, w_{k}, w_{2 k}, w_{3 k}, w_{4 k}\right)$ & $k=(n+2) / 4$ \\
\hline \multirow{2}{*}{$\mathcal{C}_{3}$} & $\left(u_{0}, u_{1}, u_{2}, u_{3}, w_{3}, w_{(n / 3)+2}, w_{(2 n / 3)+1}, w_{0}\right)$ & $k=(n / 3)-1$ \\
\cline { 2 - 4 } & $\left(u_{3}, u_{2}, u_{1}, u_{0}, w_{0}, w_{(n / 3)+1}, w_{(2 n / 3)+2}, w_{3}\right)$ & $k=(n / 3)+1$ \\
\cline { 2 - 4 } & $\left(u_{0}, u_{1}, u_{2}, u_{3}, w_{3}, w_{2}, w_{1}, w_{0}\right)$ & $k=1$ \\
\hline \multirow{2}{*}{$\mathcal{C}_{4}$} & $\left(u_{0}, u_{1}, \ldots, u_{4}, w_{4}, w_{(n+4) / 2}, w_{0}\right)$ & $k=(n / 2)-2$ \\
\cline { 2 - 4 } & $\left(u_{0}, u_{1}, \ldots, u_{4}, w_{4}, w_{2}, w_{0}\right)$ & $k=2$ \\
\hline $\mathcal{C}_{5}$ & $\left(u_{0}, u_{ \pm 1}, \ldots, u_{ \pm 5}, w_{ \pm 5}, w_{0}\right)$ & $k=(n / 2)-1$ \\
\hline $\mathcal{C}_{6}$ & $\left(u_{0}, u_{1}, w_{1}, w_{n / 2}, u_{n / 2}, u_{(n+2) / 2}, w_{(n+2) / 2}, w_{0}\right)$ & $n \geq 4$ \\
\hline $\mathcal{C}_{7}$ & $\left(u_{0}, u_{1}, w_{1}, w_{k+1}, u_{k+1}, u_{k}, w_{k}, w_{0}\right)$ & $k=n-5$ \\
\hline
\end{tabular}

Table 2: Characterization of all 8-cycles from $G(n, k)$.

We now consider the values of $s$ in turn.

\section{8-cycles with $s=0$.}

We first note that, since $G$ is large, the cycle in the outer rim cannot be of length 8 , and so the edge-set of any such cycle is a subset of $E_{I}(G)$, implying the necessary condition $n / \operatorname{gcd}(n, k)=8$. It follows that either there are no 8 -cycles with $s=0$, or there are $n / 8$ such cycles, with $k=n / 8$ or $3 n / 8$. We label these cycles $\mathcal{C}_{0}$. Also observe that these cycles (if they exist) have $(n / 8)$-fold rotational symmetry, so for each edge $e \in E_{I}(G)$, we have $\sigma(e)=1$.

\section{8-cycles with $s=2$.}

We next focus on the 8-cycles that contain two edges from $E_{S}(G)$. Any such 8-cycle contains $j$ edges in $E_{J}(G)$ and $6-j$ edges in $E_{I}(G)$, and may be rotated by the $n$-fold rotational symmetry of $G$. We further observe that $1 \leq j \leq 5$, and label these 8-cycles $\mathcal{C}_{j}$. It follows that $\left(\delta_{j}, \delta_{s}, \delta_{i}\right)=(j, 2,6-j)$. We now consider all possibilities with respect to their value $j$.

$\mathcal{C}_{1}$ When $j=1$, any corresponding cycle is of type $\left(u_{0}, w_{0}, w_{k}, w_{2 k}, w_{3 k}, w_{4 k}, w_{5 k}, u_{5 k}\right)$ if and only if $5 k \pm 1 \equiv 0(\bmod n)$.

$\mathcal{C}_{2}$ Similarly, if such an 8-cycle exists, then $4 k \equiv \pm 2(\bmod n)$, and since $k<n / 2$, it follows that $k=(n \pm 2) / 4$ or $k=(n-1) / 2$. In the former case $n \equiv 2(\bmod 4)$, and in the latter case $n$ is odd.

$\mathcal{C}_{3}$ If $j=3$, then $3 k \equiv \pm 3(\bmod n)$. So 3 divides $n$, and we have $k=1$ or $(n / 3)-1$ or $(n / 3)+1$.

$\mathcal{C}_{4}$ If $j=4$, then $2 k \equiv \pm 4(\bmod n)$. Since $k<n / 2$, there are again two possibilities: $k=2$ or $k=(n-4) / 2$.

$\mathcal{C}_{5}$ If $j=5$, then $k \equiv 5(\bmod n)$. 


\section{8-cycles with $s=4$.}

First, we consider a special type of 8-cycle of the form

$$
\left(u_{0}, u_{1}, w_{1}, w_{n / 2}, u_{n / 2}, u_{(n+2) / 2}, w_{(n+2) / 2}, w_{0}\right),
$$

whenever $n=2 k+2$. These 8 -cycles have only $(n / 2)$-fold rotational symmetry, so $\delta_{j}=1, \delta_{s}=2$, and $\delta_{i}=1$ and are labeled by $\mathcal{C}_{6}$.

Finally, consider the 8-cycles with four edges from $E_{S}(G)$ of type

$$
\left(u_{i}, w_{i}, w_{i+k}, u_{i+k}, u_{i+k+1}, w_{i+k+1}, w_{i+1}, u_{i+1}\right),
$$

where $0 \leq i \leq n-1$. Since $G$ is assumed to be large, these cycles are always present. Since they have $n$-fold symmetry, $\delta_{j}=2, \delta_{s}=4$, and $\delta_{i}=2$ and are denoted by $\mathcal{C}_{7}$.

\section{$3 \quad$ Recognizing generalized Petersen graphs}

Using the structure of 8-cycles in $G$, we have the following property.

Lemma 1. Let $G(n, k) \in \mathrm{GP}$ be a large graph, and let $\mathcal{P}$ be a partition of its edge-set, assigning edges e with the same value of $\sigma(e)$ to the same partition. Then $\mathcal{P}$ contains a part of size $n$.

Proof. Let $G=G(n, k)$. By Remark 1, it is enough to prove that $|\mathcal{P}|>1$. In addition to the cycles of type $\mathcal{C}_{7}$, more than one additional type of 8-cycle may coexist in $G$ (see existence conditions on Table 2).

We show that this can never happen in a large graph. Indeed, assume that, together with $n$ cycles of type $\mathcal{C}_{7}$, two distinct additional types of 8-cycles exist and consider the possible $\left(\begin{array}{l}7 \\ 2\end{array}\right)=21$ cases.

Case 1. There exist cycles of type $\mathcal{C}_{7}, \mathcal{C}_{0}$, and $\mathcal{C}_{1}$. Then $n \geq 4$, and $n=8 k$ or $3 n=8 k$, and $n=5 k \pm 1$ or $2 n=5 k \pm 1$. But then our graph is small.

Case 2. There exist cycles of type $\mathcal{C}_{7}, \mathcal{C}_{0}$, and $\mathcal{C}_{2}$. Then $n \geq 4$, and $n=8 k$ or $3 n=8 k$, and $n=4 k+2$ or $2 n=4 k+2$ or $k=(n+2) / 4$. Again, this implies that our graph is small.

Case 5. There exist cycles of type $\mathcal{C}_{7}, \mathcal{C}_{0}$, and $\mathcal{C}_{1}$. Then $n \geq 4$, and $n=8 k$ or $3 n=8 k$, and $k=5$ or $k=n-5$. Then we could have $G \simeq G(40,5)$. Again our graph is small.

Case 21. There exist cycles of type $\mathcal{C}_{7}, \mathcal{C}_{5}$, and $\mathcal{C}_{6}$. Then $n \geq 4$, and $k=5$ or $k=n-5$, and $k=(n / 2)-1$. But then either $G \simeq G(12,6)$ or $G \simeq G(8,3)$, which is still small.

There are at most eight distinct possibilities for the corresponding values of $\left(\sigma_{j}, \sigma_{s}, \sigma_{i}\right)$. In particular,

$$
\left(\sigma_{j}, \sigma_{s}, \sigma_{i}\right) \in\{(2,4,3),(3,6,7),(4,6,6),(5,6,5),(6,6,4),(7,6,3),(3,6,3),(2,4,2)\},
$$

where the values above are obtained by adding $(2,4,2)$ to the possible values of $\left(\delta_{j}, \delta_{s}, \delta_{i}\right)$ - see Table 1. To prove this, it is enough to observe that in the eight possible triples from (1), the values never coincide. 


\subsection{A recognition algorithm}

In this section we describe a simple procedure main $(G)$, which runs in time $O(n)$ and determines whether the input graph $G$ is a member of GP. It is described in Algorithm 2 and uses an additional procedure extend $(G, U)$ that is given in Algorithm 1 .

The tasks of these Algorithms 1 and 2 basically correspond to identifying the vertices of the outer rim, and checking whether this outer rim can be extended to a proper member of GP.

The input of Algorithm 1 consists of a connected cubic graph $G$ of order $2 n$, and the $n$ set $U$ of vertices. A procedure extend $(G, U)$ assumes that $U$ corresponds to either $E_{J}(G)$ or $E_{I}(G)$, and requires $O(n)$ time to decide whether $G \in$ GP. Indeed, once one identifies the vertices in $E_{J}(G)$ by using either $U$ or its complement (up to line 6), a bijection $V(G) \rightarrow$ $\left\{u_{0}, u_{1}, \ldots, u_{n-1}, w_{0}, w_{1}, \ldots, w_{n-1}\right\}$ is established and $k$ is easily determined (see line 11). So it is enough to check that the edges of $G$ do indeed map to the edges of $G(n, k)$.

Algorithm 2 basically consists of:

i. determining the values of $\sigma_{G}(e)$ for each edge $e \in E(G)$;

ii. determining an $n$-subset of $E(G)$ which is also a member of $\left\{E_{J}(G), E_{S}(G), E_{I}(G)\right\}$, whenever $G \in \mathrm{GP}$;

iii. identifying a vertex-set $U$ which is one of $\left\{u_{i}\right\}_{i=0}^{n-1}$ or $\left\{w_{i}\right\}_{i=0}^{n-1}$, and running extend $(G, U)$ accordingly.

We comment on these three statements in turn.

i. For any $e \in E(G)$, all 8-cycles that contain $e$ lie within its 4-neighborhood. If $G^{\prime}=$ $G\left[\cup_{i=0}^{4} N_{i}(e)\right]$, then $\sigma_{G}(e)=\sigma_{G^{\prime}}(e)$. But since $G$ is cubic, the order of $G^{\prime}$ is bounded above by 62 . So the calculation of $\sigma_{G}(e)$ takes $O(1)$ time, and the whole loop at line 2 altogether takes at most $O(|E(G)|)=O(n)$ time. In line 5, according to its value of $\sigma_{G}$, each edge is classified to the corresponding part from an edge partition $\mathcal{P}$.

ii. While line 6 is trivially of constant time-complexity, its correctness is provided by Lemma 1 Note that if $\min _{U \in \mathcal{P}}|U| \neq n$, then the same lemma allows us to return False.

[iii. If $G$ is a generalized Petersen graph, it is enough to determine a 2-factor in $G$ of order $n$, and assign it to $U$. This is trivially satisfied if the graph selected in line 6 already corresponds to either $E_{J}(G)$ or $E_{I}(G)$. If $U$ (as selected in line 6) corresponds to $E_{S}(G)$, then it is a perfect matching of order $2 n$ and the largest cycle of $G-E(U)$ clearly corresponds to $E_{J}(G)$.

\subsection{Recognizing small graphs}

It is clear that for graphs on at most 80 vertices, the membership of GP may theoretically be determined in a constant time. However, the task may not be easy in practice. We now argue that Algorithm 2 fails for precisely ten members of GP, and that it can safely be used on any graphs of order not equal to $6,8,10,16,20,24,26,48$, or 52 .

It is clear that Lemma 1 cannot hold for all small graphs. Indeed, by Frucht et al. [8], we know that there exist precisely seven pairs $(n, k)$ for which $G(n, k)$ is edge-transitive: these are $(n, k)=(4,1),(5,2),(8,3),(10,2),(10,3),(12,5)$, and $(24,5)$. In these cases all edges have the same value for $\sigma$. 
Using a computer one can easily calculate the $\sigma$-partitions for the remaining $373(n, k)$-pairs which correspond to small generalized Petersen graphs. We have checked whether the corresponding $\sigma$-partition of edges consists of more than one part and have determined that, in addition to the above seven pairs, there are three additional members of GP for which all edges touch the same number of 8-cycles. These three additional cases are $G(3,1), G(13,5)$, and $G(26,5)$. For $G(3,1)$, we trivially have $\sigma(e)=0$ for each edge $e$. The remaining two cases contain 8-cycles of types $C_{1}, C_{5}$, and $C_{7}$, and in these cases we have

$$
\left(\sigma_{j}, \sigma_{s}, \sigma_{i}\right)=(1,2,5)+(5,2,1)+(2,4,2)=(8,8,8),
$$

so $\sigma(e)=8$ for each edge $e$. It is worth noting that the three exceptional pairs correspond to vertex-transitive members of GP, which gives the following corollary.

Corollary 1. In the family of generalized Petersen graphs, there exist precisely ten members $G(n, k)$ such that all edges touch the same number of eight-cycles. These are all vertex-transitive, in particular

$$
(n, k) \in\{(3,1),(4,1),(5,2),(8,3),(10,2),(10,3),(13,5),(12,5),(24,5),(26,5)\} .
$$

It is clear that our algorithms will never recognize these ten special graphs, but will safely recognize any graph on $n$ vertices, where $n \notin\{6,8,10,16,20,24,26,48,52\}$, in $O(n)$ time.

\section{Acknowledgement}

The authors would like to thank Prof. Martin Milanič and the anonymous reviewers for their valuable comments during the preparation of this paper. The first author gratefully acknowledges the Slovenian Research Agency for funding project P1-0383 and program J1-1692, and the European Commission for funding the InnoRenew CoE project (Grant Agreement \#739574) under the Horizon2020 Widespread-Teaming program and the Republic of Slovenia (Investment funding of the Republic of Slovenia and the European Union of the European Regional Development Fund). 

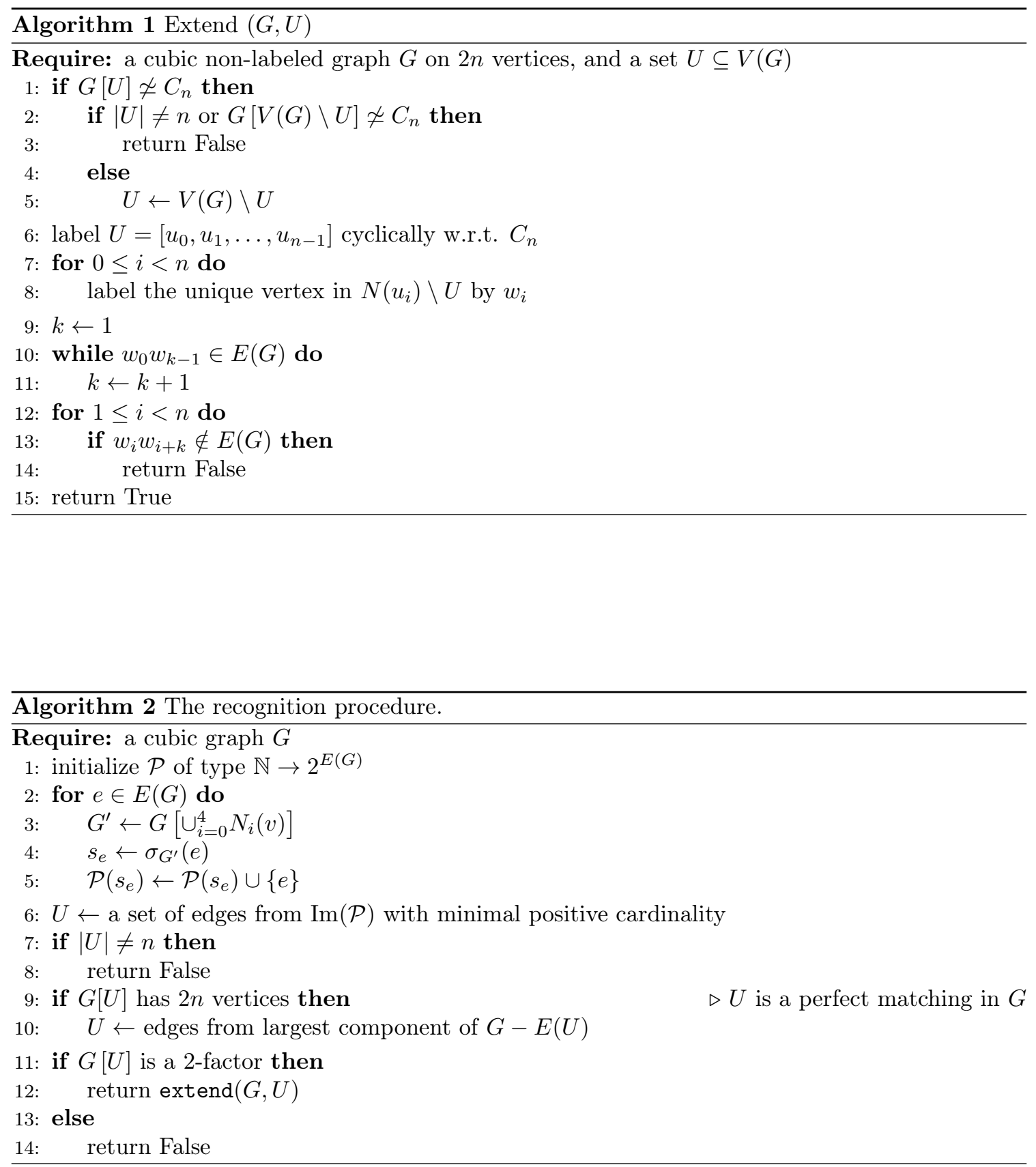


\section{References}

[1] B. Alspach, The classification of hamiltonian generalized Petersen graphs, J. Combin. Theory B 34 (1983), 293-312.

[2] B. Alspach, P. J. Robinson and M. Rosenfeld, A result on hamiltonian cycles in generalized petersen graphs, J. Combin. Theory B 31 (1981), 225-231.

[3] J. A. Bondy, Variations on the hamiltonian theme, Canad. Math. Bull 15 (1972), 57-62.

[4] G. Boruzanlı Ekinci and J. B. Gauci, On the reliability of generalized Petersen graphs, Discrete Appl. Math. 252 (2019), 2-9.

[5] F. Castagna and G. Prins, Every generalized Petersen graph has a Tait coloring, Pac. J. Math. 40 (1972), 53-58.

[6] G. Chartrand, H. Hevia and R. J. Wilson, The ubiquitous Petersen graph, Discrete Math. 100 (1992), $303-311$.

[7] H. S. Coxeter, Self-dual configurations and regular graphs, Bull. Amer. Math. Soc 56 (1950), 413-455.

[8] R. Frucht, J. E. Graver and M. E. Watkins, The groups of the generalized Petersen graphs, Proc. Cambridge Philos. Soc. 70 (1971), 211-218.

[9] D. A. Holton and J. Sheehan, The Petersen Graph, volume 7 of Australian Mathematical Society Lecture Series, Cambridge University Press, Cambridge, 1993.

[10] D. D. D. Jin and D. G. L. Wang, On the minimum vertex cover of generalized Petersen graphs, Discrete Appl. Math. 266 (2019), 309-318.

[11] S. Klavžar and A. Lipovec, Partial cubes as subdivision graphs and as generalized petersen graphs, Discrete Math. 263 (2003), 157-165.

[12] M. Krnc and T. Pisanski, Generalized Petersen graphs and Kronecker covers, Discrete Math. Theor. Comput. Sci. 21 (2019), Paper No. 15, 16.

[13] W. Lu, C. Yang and H. Ren, Lower bound on the number of Hamiltonian cycles of generalized Petersen graphs, Discuss. Math. Graph Theory 40 (2020), 297-305.

[14] R. Nedela and M. Škoviera, Which generalized Petersen graphs are Cayley graphs?, J. Graph Theory 19 (1995), 1-11.

[15] M. Petkovšek and H. Zakrajšek, Enumeration of I-graphs: Burnside does it again, Ars Math. Contemp. 2 (2009), 241-262.

[16] Y. Sakamoto, Hamilton cycles in double generalized Petersen graphs, Discuss. Math. Graph Theory 39 (2019), 117-123.

[17] M. L. Saražin, A note on the generalized Petersen graphs that are also Cayley graphs, J. Combin. Theory B 69 (1997), 226-229. 
[18] C. Tong, X. Lin and Y. Yang, Equitable total coloring of generalized Petersen graphs $P(n, k)$, Ars Combin. 143 (2019), 321-336.

[19] M. E. Watkins, A theorem on Tait colorings with an application to the generalized Petersen graphs, J. Combin. Theory 6 (1969), 152-164.

[20] Z.-B. Zhang and Z. Chen, Length of cycles in generalized Petersen graphs, Acta Math. Univ. Comenian. (N.S.) 88 (2019), 1093-1100. 